\title{
Hábitos alimentarios de Dosidicus gigas (D’ Orbigny, 1835) (Cephalopoda: Teuthoidea) frente a la costa centro-sur de Chile
}

\author{
Feeding habits of Dosidicus gigas (D’Orbigny, 1835) (Cephalopoda: \\ Teuthoidea) in the central-south coast off Chile
}

PATRICIO ULLOA ${ }^{1,3}$, MARTA FUENTEALBA $^{2} \&$ VÍCTOR RUIZ $^{3}$

\author{
${ }^{1}$ Programa Magíster en Ciencias, Mención Zoología, Escuela de Graduados, Universidad de Concepción, \\ Casilla 160-C,Concepción, Chile; e-mail: pulloa@udec.cl \\ ${ }^{2}$ Instituto de Ciencias Básicas, Universidad Católica del Maule, Casilla 160, Talca, Chile \\ e-mail:mfuentea@hualo.ucm.cl \\ ${ }^{3}$ Departamento de Zoología, Facultad de Ciencias Naturales y Oceanográficas, Universidad de Concepción, \\ Casilla 160-C,Concepción, Chile; e-mail: vruiz@udec.cl
}

\begin{abstract}
RESUMEN
Se estudiaron los hábitos alimentarios del calamar rojo Dosidicus gigas (D’Orbigny, 1835) frente a la costa de Chile centro-sur, durante el invierno del año 2005. Se examinaron un total de 52 estómagos, en todos los cuales había contenido alimentario. Los ejemplares provenían de pescas efectuadas por la flota arrastrera industrial que opera entre $36^{\circ} 39^{\prime} \mathrm{S}$ y $38^{\circ} 55^{\prime} \mathrm{S}$ sobre la merluza común Merluccius gayi (Guichenot 1848 ) y a una profundidad media de $290 \mathrm{~m}$ sobre la plataforma continental. Para la descripción del espectro trófico, alimento principal y el establecimiento de las eventuales diferencias sexuales u ontogenéticas en la frecuencia de aparición de los ítemes presa, se utilizaron los métodos numérico, gravimétrico, de frecuencia de ocurrencia, índice de importancia relativa (IIR) e índice de similitud porcentual (ISP). No se observaron diferencias sexuales ni ontogenéticas en la frecuencia de aparición de los ítemes presa. Los resultados muestran que D. gigas es un depredador oportunista, principalmente ictiófago, y secundariamente malacófago (incluye el canibalismo) y carcinófago. Se discuten los eventuales motivos e implicancias de tal conducta.
\end{abstract}

Palabras clave: Dosidicus gigas, Chile, Pacífico centro-sur, medio ambiente.

\begin{abstract}
A study on the feeding habits of the jumbo squid Dosidicus gigas (D'Orbigny, 1835) was carried out during the winter season 2005, in waters of the Chilean central and southern Pacific. A total of 52 jumbo squid stomachs from the fishing of the continental slope were collected and examined, in the Eighth and Ninth regions, between $36^{\circ} 40^{\prime} \mathrm{S}$ and $38^{\circ} 55^{\prime} \mathrm{S}$, at an average depth of $290 \mathrm{~m}$. To determine the trophic spectrum, the feeding preferences and the importance of the sex and ontogenetic condition in the diet, the numeric, gravimetric, frequency of occurrence methods, and the index of relative importance and dietary similarity were utilized. The frequency of the prey did not vary with relation to the sex and ontogenetic conditions, and was besides a hight trophic similarity in such analysis. The results showed that the diet of D. gigas consist predominatly of fishes and secondly of Mollusca (to include the cannibalism) and crustacean; suggesting that D. gigas is an oportunistic predator of third trophic level. We discuss the reasons and implications of such behavior.
\end{abstract}

Key words: Dosidicus gigas, Chile, central and south Pacific, winter feeding, environment.

\section{INTRODUCCIÓN}

Dosidicus gigas es un activo depredador en todos los estados de su ciclo de vida (Ehrhardt et al. 1983), y es conocido en Chile con los nombres de jibia o calamar rojo. Es el molusco nectónico de mayor tamaño corporal y uno de los más abundantes del Pacífico Oriental, de donde es endémico (Schmiede \& Acuña 1992, Fernández \& Vásquez 1995, Clarke \& Paliza 2000, Markaida \& Sosa-Nishizaki 2001, Nigmatullin et al. 2001).

La importancia pesquera de D. gigas en Chile ha sido irrelevante e irregular en los últimos cincuenta años (período desde que se tienen antecedentes oficiales de su captura), 
con un desembarque nacional anual promedio que no supera las 1.123 toneladas netas (Fernández \& Vásquez 1995, Rocha \& Vega 2003, Sernapesca 2003). A partir del año 2004, sin embargo, esta condición cambió drásticamente, con un desembarque anual por sobre las 416.000 toneladas netas y cuyo destino, debido al gran volumen y falta de mercado para consumo humano directo, ha sido principalmente la reducción (SERNAPESCA 2004).

Los escasos estudios sobre la alimentación de D. gigas en Chile (Wilhelm 1954, Cubillos et al. 2004) señalan que posee un amplio espectro trófico, y que su dieta está dominada por peces y secundariamente por moluscos (incluyendo el canibalismo) y crustáceos (Wilhelm 1954, Clarke \& Paliza 2000, Nigmatullin et al. 2001, Markaida \& SosaNishizaki 2003, Cubillos et al. 2004), aunque hay excepciones (Sánchez 2003). Algunos autores consideran que estos cefalópodos son especies importantes en la estructura de los ecosistemas marinos pelágicos (Markaida \& Sosa-Nishizaki 2003). En este estudio se realiza una descripción de la composición de la dieta del calamar rojo sobre la plataforma continental de Chile centro-sur. Se pretende establecer si hay variaciones de acuerdo a la condición sexual y ontogenética, y confrontar los resultados con los de otros trabajos en esta especie.

\section{MATERIALES Y MÉTODOS}

Durante el mes de julio del año 2005, se recolectaron 52 ejemplares de Dosidicus gigas, capturados en dos sitios sobre la plataforma continental de Chile $\left(36^{\circ} 44^{\prime} \mathrm{S}, 73^{\circ} 35^{\prime} \mathrm{O}\right.$ a $36^{\circ} 39^{\prime}$ 'S, $\left.73^{\circ} 34^{\prime} \mathrm{O}\right)$ y $\left(38^{\circ} 47^{\prime} \mathrm{S}, 73^{\circ} 57^{\prime} \mathrm{O}\right.$ a $\left.38^{\circ} 55^{\prime} \mathrm{S}, 73^{\circ} 52^{\prime} \mathrm{O}\right)$, a una profundidad promedio de $290 \mathrm{~m}$, por naves pertenecientes a la flota arrastrera industrial que captura la merluza común $M$. gayi. El tamaño mínimo muestral usó el criterio empírico de 20 a 25 ejemplares señalado por Shukhgalter \& Nigmatullin (2001), Markaida \& SosaNishizaki (2003) y Cubillos et al. (2004), y también, cómo se comportaba la riqueza bruta acumulada de ítemes presa en función del incremento en el tamaño de dicha muestra. Para la elección aleatoria consecutiva entre los 52 estómagos, se recurrió a tablas de números aleatorios, de acuerdo al procedimiento señalado por Cid (2004).

A cada ejemplar se le midió la longitud del manto (LM) al cm más cercano, se pesó $(0,5 \mathrm{~kg}$ de precisión) y se determinó el sexo, para luego ser extraído el estómago, los que fueron inmediatamente preservados en formalina al 10 $\%$. En el laboratorio, los estómagos fueron traspasados a alcohol al $70 \%$, para su posterior examen. La determinación taxonómica del contenido estomacal se realizó utilizando el criterio del nivel taxonómico más bajo posible, sin perjuicio de que los contenidos no identificados, fueron agrupados en categorías taxonómicas de orden superior. Para la identificación de los restos estomacales, se recurrió a bibliografía especializada tanto para peces (Wisner 1971, 1974, Rojo 1976, Sáez 1982, Leible \& Miranda 1989, Falabella et al. 1995), como moluscos (Roper et al. 1984, Markaida 2001) y crustáceos (Bahamonde 1968, Retamal 1977, 1981).

La descripción cuantitativa del contenido estomacal incluyó los siguientes métodos: (i) numérico, N (Berg 1979, Hyslop 1980), (ii) gravimétrico, G (Hyslop 1980), (iii) frecuencia de ocurrencia sobre los estómagos examinados, F (Berg 1979, Hyslop 1980) y (iv) el Índice de Importancia Relativa, IIR (Berg 1979).

La determinación de la similitud trófica entre sexos y grupos ontogenéticos se basó en el índice de Bray-Curtis, o Índice de Similitud Porcentual, ISP (Bloom 1981, González 2001). Los individuos fueron separados en dos grupos ontogenéticos (grupo de individuos de una LM $<76 \mathrm{~cm}$ y, grupo de individuos de una $\mathrm{LM} \geq \mathrm{a}$ los $76 \mathrm{~cm}$ ), bajo el criterio del tamaño promedio que alcanza esta especie cuando cumple el año de vida (sobrevida estimada), y que bordea los $76 \mathrm{~cm}$ de LM (Arguelles et al. 2001, Nigmatullin et al. 2001, Markaida et al. 2004) y en la búsqueda de un criterio biológico, que pudiera ser utilizado dentro del rango de tamaños (LM) encontrados en los individuos obtenidos en la muestra.

\section{RESULTADOS Y DISCUSIÓN}

De los 52 ejemplares muestreados, el 55,8\% $(n=29)$ correspondieron a hembras, y la razón de sexos fue de 1:0,8. La LM de las jibias 
osciló entre 59 y $89 \mathrm{~cm}$, con un tamaño medio en los machos de 79,6 cm $(\mathrm{DE}=5,87 \mathrm{~cm} ; \mathrm{n}=$ $23)$, y de $76,3 \mathrm{~cm}$ en las hembras ( $\mathrm{DE}=7,27$ $\mathrm{cm} ; \mathrm{n}=29$ ).

Se observó una alta similitud dietaria entre sexos (ISP $=96,9 \%$ ) y entre grupos ontogenéticos (ISP $=95,5 \%$ ), y por ende se encontraron diferencias no significativas en la frecuencia de aparición de los ítemes presa $\left(X^{2}\right.$ $=0,57 ; \mathrm{gl}=6 ; \mathrm{P}>0,05$ y $\mathrm{X}^{2}=6,58 ; \mathrm{gl}=7 ; \mathrm{P}>$ 0,05 , respectivamente).

Todos los estómagos tenían restos alimentarios. El espectro trófico de D. gigas estuvo compuesto por 10 ítemes presa (Tabla 1), lo que concuerda con la amplitud dietaria de registros previos (Wilhelm 1954, Ehrhardt et al. 1983, Castellanos 1994, Wormuth 1998, Markaida 2001, Markaida \& Sosa-Nishizaki 2003, Cubillos et al. 2004), y en que predominan los teleósteos (IIR $=97,7 \%)$, y entre ellos $M$. gayi $(\mathrm{IIR}=92,7 \%)$. El segundo grupo alimentario son los moluscos en donde el $99,1 \%$ de su IIR corresponde a canibalismo.
La característica depredación oportunista de D. gigas, en este caso está asociada a M. gayi, se entiende por la captura que $D$. gigas hace sobre presas abundantes, concentrada en las redes de pesca y altamente disponible (Ehrhardt et al. 1983, Clarke \& Paliza 2000, Rodhouse 2001, Anderson \& Rodhouse 2001, Markaida \& Sosa-Nishizaki 2003, Cubillos et al. 2004). Algo similar puede explicar la alta presencia de la merluza de cola Macruronus magellanicus (Lonnberg 1907) en la dieta de D. gigas observada en muestras también tomadas en la plataforma continental de Chile centro-sur (Cubillos et al. 2004). Dichas muestras provenían de la flota de cerco y arrastre de media agua, que opera principalmente sobre especies epi y mesopelágicas, tales como el jurel Trachurus symmetricus y la merluza de cola M. magellanicus. Lo anterior refuerza la idea de la conducta oportunista de D. gigas, y explica la gran asociación que existiría entre sus principales ítemes presa, y aquellos que constituyen la pesca objetivo de las naves asociadas a la toma de las muestras.

TABLA 1

Valores absolutos y porcentuales de 10 ítemes presa hallados en los estómagos de Dosidicus gigas según los métodos numérico $(\mathrm{N})$, gravimétrico $(\mathrm{G})$, de frecuencia de ocurrencia $(\mathrm{F})$ e índice de importancia relativa (IIR)

Absolute values and percent of 10 prey items found in the stomachs of Dosidicus gigas according to the numeric following methods: numerical $(\mathrm{N})$, gravimetric $(\mathrm{G})$, frequency of occurrence $(\mathrm{F})$ and index of relative importance (IIR)

\begin{tabular}{|c|c|c|c|c|c|c|c|c|}
\hline Presa & $\mathrm{N}$ & $\%$ & $\mathrm{G}(\mathrm{g})$ & $\%$ & $\mathrm{~F}$ & $\%$ & IIR & $\operatorname{IIR}(\%)$ \\
\hline Merluccius gayi & 89 & 60,54 & 5660 & 71,34 & 41 & 78,85 & 10398,74 & 92,69 \\
\hline Teleostei & 14 & 9,52 & 1103,9 & 13,91 & 9 & 17,31 & 405,57 & 3,62 \\
\hline Dosidicus gigas & 11 & 7,48 & 354,4 & 4,47 & 11 & 21,15 & 252,74 & 2,25 \\
\hline Hygophum sp. & 19 & 12,93 & 46,8 & 0,59 & 2 & 3,85 & 52,05 & 0,46 \\
\hline Helicolenus lengerichi & 3 & 2,04 & 351 & 4,42 & 3 & 5,77 & 37,27 & 0,33 \\
\hline Epigonus crassicaudus & 4 & 2,72 & 272,3 & 3,43 & 3 & 5,77 & 35,49 & 0,32 \\
\hline Caelorhynchus sp. & 4 & 2,72 & 111,1 & 1,40 & 4 & 7,69 & 31,68 & 0,28 \\
\hline Teuthoidea & 1 & 0,68 & 16,4 & 0,21 & 1 & 1,92 & 1,71 & 0,015 \\
\hline Merluccidae & 1 & 0,68 & 16,7 & 0,21 & 1 & 1,92 & 1,71 & 0,015 \\
\hline Caridea & 1 & 0,68 & 1,1 & 0,01 & 1 & 1,92 & 1,32 & 0,012 \\
\hline Total & 147 & 99,99 & $7.933,70$ & 99,99 & XX & $\mathrm{XX}$ & $11.218,28$ & 99,99 \\
\hline
\end{tabular}


El presente trabajo también ratifica la existencia del canibalismo. Dentro del grupo de los moluscos, se constituye en el principal ítem alimentario, al menos, para el grupo de tamaños de individuos estudiados y, que tanto en el Hemisferio Sur como en el Norte, concuerdan a lo menos en un $90 \%$, con los tamaños de aquellos grupos que bajo distintos criterios (e.g., reproductivos, ontogenéticos, ecológicos, conductuales, parasitológicos, evolutivos), son considerados como los ejemplares adultos de mayor talla (Ehrhardt et al. 1983, Tafur \& Rabí 1997, Argüelles et al. 2001, Markaida \& Sosa-Nishizaki 2001, Nigmatullin et al. 2001, Shukhgalter \& Nigmatullin 2001, Markaida et al. 2004). Nuestros resultados también son coincidentes con Cubillos et al. (2004), en donde D. gigas, representa un 96,8 $\%$ del IIR de las presas del Phylum Mollusca, siendo en nuestro caso del 99,3\%.

Finalmente, la ausencia de diferencias en la dieta según sexo o categoría ontogenética también ha sido documentada para esta especie. Así para el Hemisferio Norte, se ha señalado que para las presas principales, las mayores variaciones se asocian a cambios estacionales y geográficos, más que a los de tipo sexual y ontogenético (Markaida \& Sosa-Nishizaki 2003).

\section{AGRADECIMIENTOS}

Los autores agradecen la colaboración prestada por las empresas pesqueras El Golfo S.A., Bíobío S.A. y Congelados del Pacífico S.A., de Talcahuano, por las facilidades dadas para la obtención y procesamiento de las muestras en terreno. También hacemos extensivos nuestros agradecimientos a los profesores Sres. Hugo Iván Moyano, Marco Antonio Retamal y al Dr. Ciro Oyarzún de la Universidad de Concepción, por la colaboración en la determinación de algunas muestras de contenidos estomacales, al profesor Sr. Hugo Iván Moyano y a los Drs. Margarita Marchant y Juan Carlos Ortiz por la lectura crítica del manuscrito, así como a los correctores anónimos del mismo.

\section{LITERATURA CITADA}

ANDERSON C \& P RODHOUSE (2001) Life cycles, oceanography and variability: ommastrephid squid in variable oceanographic environments. Fisheries Research 54: 133-143.
ARGUELLES J, P RODHOUSE, P VILLEGAS \& G CASTILLO (2001) Age, growth and population structure of the jumbo flying squid Dosidicus gigas in Peruvian waters. Fisheries Research 54: 51-61.

BAHAMONDE N (1968) Bosquejo taxonómico sobre los estomatópodos de Chile (Crustacea, Stomatopoda). Revista Universitaria (Chile) 31: 107-120.

BERG J (1979) Discussion of methods of investigating the food of fishes, with reference to a preliminary study of the prey of Gobiusculus flavescens (Gobiidae). Marine Biology 50: 263-273.

BLOOM S (1981) Similarity indices in community studies: potential pitfalls. Marine Ecology Progress Series 5: $125-128$

CASTELLANOS Z (1994) Los invertebrados. Tomo III, primera parte -moluscos. Los celomados (excluidos artrópodos). Estudio Sigma, Buenos Aires, Argentina. 206 pp.

CID L (2004) Bioestadística. Apuntes de Bioestadística II Universidad de Concepción, Concepción, Chile. $247 \mathrm{pp}$.

CLARKE R \& O PALIZA (2000) The Humboldt Current squid Dosidicus gigas (Orbigny, 1835). Revista de Biología Marina y Oceanografía (Chile) 35: 1-39.

CUBillos L, C IBÁÑEZ, C GONZÁlEZ \& A SEPÚLVEDA (2004) Pesca de jibia (Dosidicus gigas) con red de cerco entre la V y X Región, año 2003. Informe Final. Pesca de Investigación. Instituto de Investigación Pesquera Octava Región, Talcahuano, Chile. 48 pp.

EHRHARDT N, P JACQUEMIN, F GARCÍA, G GONZÁlEZ, J LÓPEZ, J ORTIZ \& A SOLIZ (1983) On the fishery and biology of the giant squid Dosidicus gigas in the Gulf of California, Mexico. En: Caddy $\mathbf{J}$ (ed) Advances in assessment of world cephalopod resources: 35 (páginas??). FAO Fisheries Technical Paper, Rome?, Italy?.

FALABELLA F, R MELÉNDEZ \& M VARGAS (1995) Claves osteológicas para peces de Chile central: un enfoque arqueológico. Editorial Artegrama, Santiago, Chile. 208 pp.

FERNÁNDEZ F \& J VÁSQUEZ (1995) La jibia gigante Dosidicus gigas (Orbigny, 1835) en Chile: Análisis de una pesquería efímera. Estudios Oceanológicos (Chile) 14: 17-21.

GONZÁLEZ P (2001) Biología reproductiva y conducta trófica de Pinguipes chilensis, Valenciennes, 1833 (Perciformes: Pinguipedidae). Tesis de Magíster, Facultad de Ciencias Biológicas y de Recursos Naturales, Universidad de Concepción, Concepción, Chile. 158 pp.

HYSLOP E (1980) Stomach contents analysis - a review of methods and their application. Journal Fish Biology 17: 411-429.

LEIBLE M \& P MIRANDA (1989) El otolito sagitta en el reconocimiento de diferentes especies de teleósteos de la costa central de Chile. Boletín de la Sociedad de Biología de Concepción (Chile) 60: 149-160.

MARKAIDA U (2001) Biología del calamar gigante Dosidicus gigas Orbigny, 1835 (Cephalopoda: Ommastrephidae) en el Golfo de California, México. Tesis Doctoral, Centro de Investigación Científica y de Educación Superior de Ensenada, Ensenada, Baja California, México. 387 pp.

MARKAIDA U \& O SOSA-NISHIZAKI (2001) Reproductive biology of jumbo squid Dosidicus gigas in the Gulf of California, 1995-1997. Fisheries Research 54: 63-82.

MARKAIDA U \& O SOSA-NISHIZAKI (2003) Food and feeding habits of jumbo squid Dosidicus gigas 
(Cephalopoda: Ommastrephidae) from the Gulf of California, Mexico. Journal of the Marine Biological Association of the United Kingdom 83: 4162.1-4162.16.

MARKAIDA U, C QUIÑÓNEZ-VELÁZQUEZ \& O SOSA-NISHIZAKI (2004) Age, growth and maturation of jumbo squid Dosidicus gigas (Cephalopoda: Ommastrephidae) from the Gulf of California, Mexico. Fisheries Research 66: 31-47.

NIGMATULLIN CH, KN NESIS \& AI ARKHIPKIN (2001) A review of the biology of the jumbo squid Dosidicus gigas (Cephalopoda: Ommastrephidae). Fisheries Research 54: 9-19.

RETAMAL M (1977) Los crustáceos decápodos chilenos de importancia económica. Gayana Zoología (Chile) 39: 1-48.

RETAMAL M (1981) Catálogo ilustrado de los crustáceos decápodos de Chile. Gayana Zoología (Chile) 44: 1110 .

ROCHA F \& M VEGA (2003) Overview of cephalopod fisheries in Chilean waters. Fisheries Research 60: 151-159.

RODHOUSE P (2001) Managing and forecasting squid fisheries in variable environments. Fisheries Research 54: 3-8.

ROJO A (1976) Osteología de la merluza argentina (Merluccius hubbsi, Marini 1933). Boletín Instituto Español de Oceanografía 219: 1-61.

ROPER C, M SWEENEY \& C NAUEN (1984) FAO species catalogue. Volume 3. Cephalopods of the world: an annotated and illustrated catalogue of species of interest to fisheries. FAO Fisheries Synopsis, Rome, Italy. $277 \mathrm{pp}$

SÁEZ L (1982) Myctophidae (Pisces, Osteichthyes, Myctophiformes) recolectado por la expedición "Itzumi-Pelágico I". Enero- Febrero 1980. Tesis de Título, Facultad de Ciencias Biológicas y de Recursos Naturales, Universidad de Concepción, Concepción, Chile. 100 pp.

SÁNCHEZ P (2003) Cephalopods from off the Pacific coast of Mexico: biological aspects of the most abundant species. Scientia Marina 67: 81-90.

SERNAPESCA (2003) Anuario estadístico de Pesca. Servicio Nacional de Pesca, Ministerio de
Economía, Fomento y Reconstrucción, Sistemas de Información y Estadísticas Pesqueras, Gobierno de Chile, Valparaíso, Chile. $156 \mathrm{pp}$

SERNAPESCA (2004) Anuario estadístico de Pesca. Servicio Nacional de Pesca, Ministerio de Economía, Fomento y Reconstrucción, Sistemas de Información y Estadísticas Pesqueras, Gobierno de Chile, Valparaíso, Chile. 181 pp.

SCHMIEDE P \& E ACUÑA (1992) Regreso de las jibias (Dosidicus gigas) a Coquimbo. Revista Chilena de Historia Natural 65: 389-390.

SHUKHGALTER O \& CH NIGMATULLIN (2001) Parasitic helminths of jumbo squid Dosidicus gigas (Cephalopoda: Ommastrephidae) in open waters of the central east Pacific. Fisheries Research 54: 95110 .

TAFUR R \& M RABÍ (1997) Reproduction of the jumbo flying squid, Dosidicus gigas (Orbigny, 1835) (Cephalopoda: Ommastrephidae) off Peruvian coast. Scientia Marina 61: 33-37.

WILHELM O (1954) Algunas observaciones acerca de las mortandades de jibias (Dosidicus gigas D'Orb.) en el litoral de Concepción. Revista de Biología Marina (Chile) 4: 196-201.

WISNER R (1971) Descriptions of eight new species of myctophid fishes from the eastern Pacific Ocean. Copeia 1: 39-54.

WISNER R (1974) The taxonomy and distribution of lanternfishes (Family Myctophidae) of the eastern Pacific Ocean. NORDA Report 3. Navy Ocean Research and Development Activity, St. Louis, Missouri, USA. 229 pp.

WORMUTH J (1998) Workshop deliberations on the Ommastrephidae: a brief history of their systematics and a review of the systematics, distribution, and biology of the genera Martialia Rochebrune and Mabille, 1899, Todaropsis Girard, 1890, Dosidicus Steenstrup, 1857, Hyaloteuthis Gray, 1849, and Eucleoteuthis Berry, 1916. En: Voss N, M Vecchione, R Toll \& M Sweeney (eds) Systematic and biogeography of cephalopods. Volumen II. Smithsonian Contributions to Zoology 586: 373-383. 
